\title{
Superconvergence of Variational Discretization for Bilinear Elliptic Optimization Problems
}

\author{
Yuelong Tang* and Yuchun Hua \\ College of Science, Hunan University of Science and Engineering, Youngzhou 425100, China \\ ${ }^{*}$ Corresponding author
}

\begin{abstract}
In this paper, variational discretization approximation of bilinear elliptic optimal control problems is considered. Firstly, we construct a variational discretization method for the bilinear elliptic optimal control problem with control constraints. Secondly, we derive the convergence of the approximation scheme. Thirdly, we analyze the superconvergence. Finally, we present a numerical example to conform our theoretical results.
\end{abstract}

Keywords-convergence; superconvergence; variational discretization; bilinear elliptic optimal control problems

\section{INTRODUCTION}

There has been so extensive research on convergence and super convergence analysis of finite element method for elliptic optimization that it is impossible to given a very brief review here. Convergence and super convergence for finite element approximation of linear or semi-linear elliptic optimization problems are investigated in [1, 2] and [3, 4], respectively.

In recent years, much research has begun to examine finite element approximations of bilinear optimal control problems. A priori error estimates of finite element discretization for bilinear elliptic optimal control problems is established in [5]. Super convergence of finite element method for optimal control problems of bilinear type is obtained in [6].

Hinze present a vatiational discretization (VD) concept for control constrained optimization problems in [7]. Its main feature is not directly to discretize the space of admissible controls but to obtain the control by utilizing the discretization of the state and co-state variables and the implicity solution of variational inequality. It can improve the convergent order from $\mathrm{O}(h)$ to $\mathrm{O}\left(h^{2}\right)$. Recent years, VD are used to solve different kinds of constrained. In this paper, we consider the VD approximation of bilinear elliptic optimization problems with control constraints.

We are interested in the following optimization problem:

$$
\min _{u \in K} \frac{1}{2} \int_{\Omega}\left(\left|y(x)-y_{d}(x)\right|^{2}+|u(x)|^{2}\right) d x
$$

subject to

$$
-\operatorname{div}(A(x) \nabla y(x)+u(x) y(x)=f(x), \quad x \in \Omega,
$$

$$
y(x)=0, \quad x \in \partial \Omega,
$$

where the set of admissible control is defined by

$$
K=\left\{v(x) \in L^{2}(\Omega): a \leq v(x) \leq b\right\}
$$

and $0<a<b<+\infty$ are constants.

Let $\Omega \subset \mathbb{R}^{d}(d=1,2,3)$ be a bounded open domain with a smooth boundary $\partial \Omega$. The coefficient $A$ is a symmetric matrix such that $\xi^{T} A \xi \geq c\|\xi\|^{2}, \xi \in \mathbb{R}^{d}$ with $c>0$. In this paper, we use the standard notation $W^{m, q}(\Omega)$ for Sobolev spaces on $\Omega$ with standard norm $\|\cdot\|_{W^{m, q}(\Omega)}$ and seminorm $|\cdot|_{W^{m, q}(\Omega)} \cdot$

\section{VD APPROXIMATION FOR THE MODEL PROBLEM}

In this section, we present a VD approximation for the optimization problem (1.1)-(1.4). For ease of exposition, we set $W_{0}^{m, q}(\Omega) \equiv\left\{v \in W^{m, q}(\Omega):\left.v\right|_{\partial \Omega}=0\right\}, H^{1}(\Omega)=W^{m, 2}(\Omega)$ and $H_{0}^{1}(\Omega)=W_{0}^{m, 2}(\Omega)$. Furthermore, we set $U=L^{2}(\Omega)$ and $W=H_{0}^{1}(\Omega)$ and denote $\|\cdot\|_{H^{m}(\Omega)}$ and $\|\cdot\|_{L^{2}(\Omega)}$ by $\|\cdot\|_{m}$ and $\|\cdot\|$, respectively.

Let

$$
\begin{aligned}
& a(v, w)=\int_{\Omega}(A \nabla v) \cdot \nabla w, \quad \forall v, w \in W, \\
& \left(f_{1}, f_{2}\right)=\int_{\Omega} f_{1} \cdot f_{2}, \quad \forall f_{1}, f_{2} \in U .
\end{aligned}
$$

It follows from the assumption on $A$, we have that

$$
\begin{aligned}
& a(v, v) \geq c\|v\|_{1}^{2}, \quad \forall v \in W, \\
& a(v, w) \leq C\|v\|_{1}\|w\|_{1}, \quad \forall v, w \in W .
\end{aligned}
$$

Then a weak formula for the model problem reads: 


$$
\left\{\begin{array}{l}
\min _{u \in K} \frac{1}{2}\left\{\left\|y-y_{d}\right\|^{2}+\|u\|^{2}\right\} \\
a(y, w)+(u y, w)=(f, w), \quad \forall w \in W .
\end{array}\right.
$$

It is well known (see, e.g., [8]) that the control problem (5) has a solution $(y, u)$, and that a pair $(y, u)$ is the solution of (5), then there is a co-state $p \in W$ such that the triplet $(y, p, u)$ satisfies the following optimality conditions:

$$
\begin{gathered}
a(y, w)+(u y, w)=(f, w), \quad \forall w \in W, \\
a(q, p)+(u p, q)=\left(y-y_{d}, q\right), \quad \forall q \in W, \\
(u-y p, v-u) \geq 0, \quad \forall v \in K .
\end{gathered}
$$

As in [9], it is easy to prove the following lemma:

Lemma 2.1 Let $(y, p, u)$ be the solution of (6)-(8). Then

$$
u=\min (\max (a, y p), b)
$$

Let $T^{h}$ and $h$ be regular triangulations of $\Omega$ and the mesh size, respectively. Moreover, we set

$$
W_{h}=\left\{v_{h} \in C(\bar{\Omega}):\left.v_{h}\right|_{\tau} \in P_{1}, \forall \tau \in T^{h},\left.v_{h}\right|_{\partial \Omega}=0\right\},
$$

Where $P_{1}$ denotes polynomials of total degree no more than 1.Thus a VD approximation of (6)-(8) is as follows:

$$
\left\{\begin{array}{l}
\min _{u_{h} \in K} \frac{1}{2}\left\{\left\|y-y_{d}\right\|^{2}+\left\|u_{h}\right\|^{2}\right\} \\
a\left(y_{h}, w_{h}\right)+\left(u_{h} y_{h}, w_{h}\right)=\left(f, w_{h}\right), \quad \forall w_{h} \in W_{h} .
\end{array}\right.
$$

The problem (10) again has a solution $\left(y_{h}, u_{h}\right)$ and that if $\left(y_{h}, u_{h}\right) \in W_{h} \times K$ is the solution of (10), then there is a costate $p_{h} \in W_{h}$, such that the triplet $\left(y_{h}, p_{h}, u_{h}\right)$ satisfies the following optimality conditions:

$$
\begin{array}{r}
a\left(y_{h}, w_{h}\right)+\left(u_{h} y_{h}, w_{h}\right)=\left(f, w_{h}\right), \quad \forall w_{h} \in W_{h}, \\
a\left(q_{h}, p_{h}\right)+\left(u_{h} p_{h}, q_{h}\right)=\left(y_{h}-y_{d}, q_{h}\right), \quad \forall q_{h} \in W_{h},
\end{array}
$$

$$
\left(u_{h}-y_{h} p_{h}, v-u_{h}\right) \geq 0, \quad \forall v \in K .
$$

Similar to the Lemma 2.1, we can easily derive the following lemma:

Lemma 2.2 Let $\left(y_{h}, p_{h}, u_{h}\right)$ be the solution of (11)-(13). Then, we have

$$
u_{h}=\min \left(\max \left(a, y_{h} p_{h}\right), b\right)
$$

Remark 2.1 We minimize over the infinite dimensional set $K$ instead of minimizing over a finite dimensional subset of $K_{\text {in (13). Then we just need to solve the discrete equations }}$ (11)-(12) and obtain $u_{h}$ from (14).

\section{ERROR ESTIMATES OF INTERMEDIATE VARIABLES}

Some intermediate variables and useful error estimation are introduced in this section. For any $v \in K, w_{h}, q_{h} \in W_{h}$, let $y_{h}(v), p_{h}(v) \in W_{h}$ satisfies the following system:

$$
a\left(y_{h}(v), w_{h}\right)+\left(v y_{h}(v), w_{h}\right)=\left(f, w_{h}\right)
$$

$$
a\left(q_{h}, p_{h}(v)\right)+\left(v p_{h}(v), q_{h}\right)=\left(y_{h}(v)-y_{d}, q_{h}\right)
$$

If $\left(y_{h}, p_{h}, u_{h}\right)$ be the solution of (11)-(13) then we have $\left(y_{h}, p_{h}\right)=\left(y_{h}\left(u_{h}\right), p_{h}\left(u_{h}\right)\right)$.

The elliptic projection operator $R_{h}: W \rightarrow W_{h}$ defined as follows: for any $\phi \in W$ satisfies

$$
a\left(\phi-R_{h} \phi, w_{h}\right)=0, \quad \forall w_{h} \in W_{h} .
$$

It has the following approximation property (see e.g., [10])

$$
\left\|\varphi-R_{h} \varphi\right\|_{s} \leq C h^{2-s}\|\varphi\|_{2}, \forall \varphi \in H^{2}(\Omega), s=0,1 .
$$

Lemma 3.1 Let $(y, p, u)$ be the solution of (6)-(8) and $\left(y_{h}(u), p_{h}(u)\right)$ be the solution of (15)-(16) with $v=u$. Suppose $u \in L^{\infty}(\Omega)$ and $y, p \in H^{2}(\Omega)$, then we have

$$
\left\|y_{h}(u)-y\right\|+\left\|p_{h}(u)-p\right\| \leq C h^{2} .
$$


Prof. Set $v=u$ in (15), then from (6) and the definition of the elliptic projection operator $R_{h}$, we have

$$
\begin{aligned}
& a\left(y_{h}(u)-R_{h} y, w_{h}\right)+\left(u\left(y_{h}(u)-R_{h} y\right), w_{h}\right) \\
= & \left(u\left(y-R_{h} y\right), w_{h}\right), \quad \forall w_{h} \in W_{h} .
\end{aligned}
$$

Note that $u \in K \cap L^{\infty}(\Omega)$ and

$a\left(y_{h}(u)-R_{h} y, y_{h}(u)-R_{h} y\right) \geq c\left\|y_{h}(u)-R_{h} y\right\|$.

From (17) and (19)-(20), we obtain

$$
c\left\|y_{h}(u)-R_{h} y\right\| \leq C h^{2}\|y\|_{2} .
$$

Hence, from (17) and (21), we derive

$$
\begin{gathered}
\left\|y_{h}(u)-y\right\| \leq\left\|y_{h}(u)-R_{h} y\right\|+\left\|R_{h} y-y\right\| \\
\leq C h^{2}\|y\|_{2} .
\end{gathered}
$$

By choosing $v=u$ in (16), then from (7) and the definition of the elliptic projection operator, we get

$$
\begin{aligned}
& a\left(q_{h}, p_{h}(u)-R_{h} p\right)+\left(u\left(p_{h}(u)-R_{h} p\right), q_{h}\right) \\
= & \left(u\left(p-R_{h} p\right), q_{h}\right)+\left(y_{h}(u)-y, q_{h}\right), \forall w_{h} \in W_{h} .
\end{aligned}
$$

Similarly, we can derive

$$
c\left\|p_{h}(u)-p\right\| \leq C h^{2}\left(\|p\|_{2}+\|y\|_{2}\right) .
$$

Then (18) follows from (22) and (24).

\section{CONVERgEnCE ANALYsis}

In this section, we will give the convergence analysis for the control variable. Just for ease of exposition, we set

$$
\begin{aligned}
& J(u)=\frac{1}{2}\left\{\left\|y-y_{d}\right\|^{2}+\|u\|^{2}\right\}, \\
& J_{h}\left(u_{h}\right)=\frac{1}{2}\left\{\left\|y_{h}-y_{d}\right\|^{2}+\left\|u_{h}\right\|^{2}\right\} .
\end{aligned}
$$

It can be shown that

$$
J^{\prime}(u)=(u-y p, u)
$$

$$
J_{h}^{\prime}\left(u_{h}\right)=\left(u_{h}-y_{h}\left(u_{h}\right) p_{h}\left(u_{h}\right), u_{h}\right) .
$$

In many applications, the objective functional $J(\cdot)$ is uniform convex near the solution $u$ (see e.g., [11]). As in [12], we assume that there is a positive constant $C$, such that

$C\|u-v\|^{2} \leq\left(J_{h}^{\prime}(u)-J_{h}^{\prime}(v), u-v\right), \quad \forall u, v \in K$

Theorem 4.1 Let $(y, p, u)$ and $\left(y_{h}, p_{h}, u_{h}\right)$ be the solutions of (6)-(8) and (11)-(13), respectively. Assume that $y_{h}(u), p \in L^{\infty}(\Omega)$ and all the conditions in the Lemma 3.1 are valid. Then, we have

$$
\left\|u-u_{h}\right\| \leq C h^{2}
$$

Prof. Set $v=u_{h}$ and $v=u$ in (8) and (13), respectively. We obtain

$$
\left(u-y p, u_{h}-u\right) \geq 0
$$

and

$$
\left(u_{h}-y_{h} p_{h}, u-u_{h}\right) \geq 0 .
$$

From (25)-(27) and (29)-(30), we have

$$
\begin{aligned}
& C\left\|u-u_{h}\right\|^{2} \\
\leq & \left(J_{h}^{\prime}(u)-J_{h}^{\prime}\left(u_{h}\right), u-u_{h}\right) \\
= & \left(u-y_{h}(u) p_{h}(u)-u_{h}+y_{h}\left(u_{h}\right) p_{h}\left(u_{h}\right), u-u_{h}\right) \\
\leq & \left(\left(y-y_{h}(u)\right) p+y_{h}(u)\left(p-p_{h}(u)\right), u-u_{h}\right) \\
\leq & C(\varepsilon)\left(\left\|y-y_{h}(u)\right\|^{2}+\left\|p-p_{h}(u)\right\|^{2}\right) \\
& +2 \varepsilon\left\|u-u_{h}\right\|^{2} .
\end{aligned}
$$

Let $\varepsilon$ be small enough, then (28) follows from (18) and (31).

\section{SUPER CONVERGENCE ANALYSIS}

In this section, we will analyze the super convergence of the state and co-state variables.

Theorem 5.1 Let $(y, p, u)$ and $\left(y_{h}, p_{h}, u_{h}\right)$ be the solutions of (6)-(8) and (11)-(13), respectively. Suppose all the conditions in the Theorem 4.1 hold. We have 


$$
\left\|y_{h}-R_{h} y\right\|_{1}+\left\|p_{h}-R_{h} p\right\|_{1} \leq C h^{2} .
$$

Prof. From (6) and (11), for any $w_{h} \in W_{h}$, we get

$$
\begin{aligned}
& a\left(y-y_{h}, w_{h}\right)+\left(u\left(y-y_{h}\right), w_{h}\right) \\
= & \left(y_{h}\left(u_{h}-u\right), w_{h}\right) .
\end{aligned}
$$

According to the definition of $R_{h}$, we obtain

$$
\begin{aligned}
& a\left(R_{h} y-y_{h}, w_{h}\right)+\left(u\left(R_{h} y-y_{h}\right), w_{h}\right) \\
= & \left(u\left(R_{h} y-y_{h}\right), w_{h}\right)+\left(y_{h}\left(u_{h}-u\right), w_{h}\right)
\end{aligned}
$$

Selecting $w_{h}=R_{h} y-y_{h}$, (17), (28) and (34), we derive

$$
\left\|R_{h} y-y_{h}\right\|_{1} \leq C h^{2}
$$

From (7) and (12), for any $q_{h} \in W_{h}$, we have

$$
\begin{aligned}
& a\left(q_{h}, p-p_{h}\right)+\left(u\left(p-p_{h}\right), q_{h}\right) \\
= & \left(p_{h}\left(u_{h}-u\right), q_{h}\right)+\left(y-y_{h}, q_{h}\right) .
\end{aligned}
$$

Similarly, we can derive

$$
\left\|R_{h} p-p_{h}\right\|_{1} \leq C h^{2}
$$

Hence, (32) follows from (35) and (37).

\section{NUMERICAL EXPERIMENT}

Let $\Omega=[0,1] \times[0,1], c=0, b=1$ and $A$ is an unit matrix.

We solve the following optimal control problem where codes developed based on AFEPack which is freely available.

Example. The data are as follows:

$$
\begin{aligned}
& y(x)=\sin \left(2 \pi x_{1}\right) \sin \left(2 \pi x_{2}\right) \\
& p(x)=y(x) \\
& u(x)=\min (\max (-0.5, y(x) p(x)), 0.5) \\
& f(x)=-\operatorname{div}(A \nabla y(x))+u(x) y(x) \\
& y_{d}(x)=y(x)+\operatorname{div}(A \nabla p(x))-u(x) p(x)
\end{aligned}
$$

The errors $\left\|u-u_{h}\right\|,\left\|y_{h}-R_{h} y\right\|_{1},\left\|p_{h}-R_{h} p\right\|_{1}$ based on a sequence of uniform refined meshes are shown in table I.
TABLE I. THE ERRORS ON UNIFORM MESH

\begin{tabular}{|l|l|l|l|}
\hline \multirow{2}{*}{$\begin{array}{c}\text { Mesh } \\
\text { nodes }\end{array}$} & $\left\|u-u_{h}\right\|$ & $\left\|y_{h}-R_{h} y\right\|_{1}$ & $\left\|p_{h}-R_{h} p\right\|_{1}$ \\
\cline { 2 - 4 } & $\| .97429 \mathrm{e}-02$ & $8.36251 \mathrm{e}-03$ & $7.42735 \mathrm{e}-03$ \\
\hline 100 & $1.31232 \mathrm{e}-02$ & $2.30218 \mathrm{e}-03$ & $2.00054 \mathrm{e}-03$ \\
\hline 400 & $3.31625 \mathrm{e}-03$ & $5.91429 \mathrm{e}-04$ & $5.10980 \mathrm{e}-04$ \\
\hline 1600 & $8.31263 \mathrm{e}-04$ & $1.48953 \mathrm{e}-04$ & $1.28457 \mathrm{e}-04$ \\
\hline 6400 & &
\end{tabular}

The superconvergence phenomenon can be observed clearly from the Table I.

\section{ACKNOWLEDGMENT}

The first author is supported by the National Natural Science Foundation of China (11401201), the project of Hunan Province Education Department (16B105), and the construct program of the key discipline in Hunan University of Science and Engineering.

\section{REFERENCES}

[1] Falk, Approximation of a class of optimal control problems with order of convergence estimates, J. Math. Anal. Appl. 44 (1973) 28-47.

[2] T. Geveci, On the approximation of the solution of an optimal control problem governed by an elliptic equation, RAIRO Anal. Numer. 13 (1979) 313-328.

[3] C. Meyer and A. Rosch, Superconvergence properties of optimal control problems, SIAM J. Control Optim. 43(3) (2004) 970-985.

[4] Y. Chen and Y. Dai, Superconvergence for optimal control problems governed by semi-linear elliptic equations, J. Sci. Comput. 39 (2009) 206-221.

[5] A. Kroner and B. Vexler, A priori error estimates for elliptic optimal control problems with a bilinear state equation, J. Comput. Appl. Math. 230(2009)781-802.

[6] D. Yang, Y. Chang and W. Liu, A priori error estimate and super convergence analysis for an optimal control problem of bilinear type, J. Comput. Math.26(4) (2008) 471-487.

[7] M. Hinze, A variational discretization concept in control constrained optimization: the linear-quadratic case, Comput. Optim. Appl. 30 (2005) 45-61.

[8] J. Lions, Optimal Control of Systems Governed by Partial Differential Equations, Springer-Verlag, Berlin, 1971.

[9] M. Hinze, N. Yan and Z. Zhou, Variational discretization for optimal control governed by convection dominated diffusion equations, J. Comput. Math. 27(2-3) (2009) 237-253.

[10] Y. Chen and Y. Dai, Superconvergence for optimal control problems governed by semi-linear elliptic equations, J. Sci. Comput. 39 (2009) 206-221.

[11] W. Liu and D. Tiba, Error estimates for the finite element approximation of a class of nonlinear optimal control problems, J. Numer. Funct. Optim. 22 (2001), 953-972.

[12] E. Casas and F. Troltzsch, Second-order necessary and sufficient optimality conditions for optimization problems and applications to control theory, SIAM J. Control Optim. 13(2) (2002) 406-431. 